\title{
Longitudinal sound mode of a Bose-Einstein condensate in an optical lattice
}

\author{
J.-P. Martikainen* and H. T. C. Stoof ${ }^{\dagger}$ \\ Institute for Theoretical Physics, Utrecht University, Leuvenlaan 4, 3584 CE Utrecht, The Netherlands
}

(Received 29 August 2003; published 12 February 2004)

\begin{abstract}
We examine the effect of the transverse breathing mode on the longitudinal sound propagation in a BoseEinstein condensate in a one-dimensional optical lattice. In particular, we discuss how the coupling with the transverse breathing mode influences the sound velocity in an optical lattice. Using a variational approach we calculate the dispersion relations for the longitudinal sound mode and the transverse breathing mode analytically, and find that the shift in the sound velocity from the uncoupled result can be large enough to be experimentally relevant. We also find that the effective mass of the transverse breathing mode is affected considerably by the coupling to longitudinal sound.
\end{abstract}

DOI: 10.1103/PhysRevA.69.023608

PACS number(s): 03.75.Hh, 32.80.Pj, 03.65.-w

\section{INTRODUCTION}

Repulsive contact interactions between atoms in a dilute Bose gas cause a qualitative change in the low-energy excitations of the system. In particular, the noninteracting singleparticle dispersion equal to $\hbar^{2} k^{2} / 2 m$ is replaced with the sound dispersion $c k$ that is linear in the momentum [1]. The existence of this sound mode is important as it allows for the possibility of superfluid flow [2]. Sound propagation in a trapped Bose-Einstein condensate was studied experimentally by Andrews et al. [3,4] and Stamper-Kurn et al. [5] in the regime where the condensate can be considered as being homogeneous in one direction. The experimental observations were in good agreement with theoretical predictions in that case $[6-8]$.

Bose-Einstein condensates can also be placed in an optical lattice. The periodic structure of the lattice has enabled studies of Bloch oscillations [9], number squeezing [10], collapses and revivals [11], and a number of superfluid properties of Bose gases [12,13]. Importantly, the Bose-Einstein condensates in an optical lattice realize the Bose-Hubbard model [14] and can be used to study the quantum phase transition from the superfluid into a Mott-insulator state [1416].

Apart from these phenomena, sound propagation is also possible in a Bose-Einstein condensate in an optical lattice. There exists several theoretical calculations for the sound velocity of a Bose-Einstein condensate in a one-dimensional optical lattice [16-21]. All these theories for the sound mode are based on the idea that the condensate wave function in site $n$ is, in tight-binding approximation, essentially one dimensional and has the form $\Phi_{n}(x, y, t)$ $=\psi(x, y) \sqrt{N_{n}(t)} e^{i \nu_{n}(t)}$. This implies that the only dynamical variables of these theories are the total number of atoms $N_{n}(t)$ and the global phase $\nu_{n}(t)$ in every site, but there is no time dependence in the wave function describing the transverse directions. Physically, it is not a priori clear that this is a valid assumption because in the presence of repulsive interactions between the atoms the profile $\psi(x, y)$ does not

\footnotetext{
*Electronic address: J.P.J.Martikainen@phys.uu.nl

${ }^{\dagger}$ Electronic address: stoof@phys.uu.nl
}

remain constant as the number of particles in the site is changed. For example, increasing the number of atoms from its equilibrium value causes the condensate to expand whereas a reduction of the number of atoms causes the condensate to contract. Therefore, number fluctuations in every site of the lattice are coupled to collective modes of the condensate in each site.

As far as we know, the coupling between the sound mode and the collective modes is absent in all the existing theories except in the recent work by Taylor and Zaremba [22], whose formulation in principle contains such a coupling although the effect is neglected in all applications discussed by these authors. In a one-dimensional optical lattice where the lattice runs in the longitudinal direction (the $z$ direction), the coupling predominantly excites the breathing mode in the transverse direction (the $x y$ plane). These transverse modes were studied previously in Ref. [21], in the approximation that the coupling with longitudinal sound can be neglected. While a coupling definitely exists between the sound mode and the transverse breathing mode, a coupling between the transverse quadrupole modes and the sound mode does not exist because these modes are orthogonal. Therefore, the results in Ref. [21] for the quadrupole modes are not affected by the coupling with the sound mode.

In this paper we go beyond the approximation used in Refs. [16-21], by providing a unified theory of longitudinal sound and the transverse breathing mode in a onedimensional optical lattice. Using a variational approach we obtain the magnitude of the change to the sound velocity when the coupling with the transverse breathing mode is taken into account. It turns out that the relative shift in the sound velocity from the uncoupled result approaches a constant $\sqrt{3 / 4}-1 \approx-0.13$ as the strength of the interactions is increased, i.e., in the Thomas-Fermi limit. Therefore, the shift is rather large and should be taken into account when quantitative results are required. Likewise the shift in the effective mass of the transverse breathing mode from the result in Ref. [21] also turns out to be large and should also be taken into account.

Intriguingly, our approach leads to a very simple theory of Josephson oscillations coupled to just one transverse degree of freedom. Such a theory has some analogies with the theories describing damped relative phase dynamics of weakly 
coupled condensates $[23,24]$. In these theories the damping of the relative phase is caused by the coupling into quasiparticles. Our minimal theory can be used to study the phase dynamics of the Bose-Einstein condensate in a onedimensional optical lattice and with considerable modifications even the (quasi-) irreversible damped phase dynamics.

This paper is organized as follows. In Sec. II, we outline the theoretical foundation of our work and explain the variational ansatz we use. In Sec. III we write down the equations of motion and solve for the eigenmodes. We then proceed to discuss, in Secs. III A and III B, the dispersion relations for the sound and the transverse breathing modes separately. Previous experimental studies of the condensate sound mode $[3,4]$ tracked the time evolution of a density dip. Therefore in Sec. IV, we apply our theory to solve numerically the time evolution of such a dip in the condensate density in an optical lattice. In addition, in Sec. IV we also solve the time evolution of the system that was prepared with a localized breathing-mode disturbance. We conclude with a brief discussion of our results in Sec. V.

\section{VARIATIONAL ANSATZ AND LAGRANGIAN}

The theory we use is similar to the one presented elsewhere [21]. In this paper the only difference with the theory presented in Ref. [21] is the use of the grand-canonical Hamiltonian, i.e., inclusion of the chemical potential term $-\mu \hat{N}$ into the energy functional. Due to the presence of the global phase factors and the atom-number fluctuations in different sites, this turns out to be more convenient for our purposes.

We consider a Bose-Einstein condensate trapped by a harmonic trap with a radial trapping frequency $\omega_{r}$. The longitudinal trapping frequency $\omega_{z} \ll \omega_{r}$ is assumed to be so small as to be irrelevant. The Bose-Einstein condensate also experiences a one-dimensional optical lattice in the longitudinal direction and this lattice splits the condensate into a stack of weakly coupled two-dimensional condensates. Furthermore, we use trap units, i.e., the unit of energy is $\hbar \omega_{r}$, the unit of time is $1 / \omega_{r}$, and the unit of length is $l_{r}=\sqrt{\hbar / m \omega_{r}}$, where $m$ is the atomic mass. The energy functional for the stack of two-dimensional condensates is then

$$
\begin{aligned}
E\left[\Phi^{*}, \Phi\right]= & \sum_{n} \int d^{2} r\left\{-\frac{1}{2} \Phi_{n}^{*}(x, y) \nabla^{2} \Phi_{n}(x, y)\right. \\
& +\left[\frac{1}{2}\left(x^{2}+y^{2}\right)+\frac{U_{2 D}}{2}\left|\Phi_{n}(x, y)\right|^{2}-\mu\right] \\
& \times\left|\Phi_{n}(x, y)\right|^{2} \\
& \left.-J \sum_{\langle n, m\rangle} \int d^{2} r \Phi_{m}^{*}(x, y) \Phi_{n}(x, y)\right\},
\end{aligned}
$$

where the lattice sites are labeled by $n$ and $\langle n, m\rangle$ indicates nearest neighbors. This energy functional is characterized by two tunable parameters: the strength of the interaction $U_{2 D}$ in every two-dimensional Bose-Einstein condensate and the strength $J$ of the Josephson coupling between the condensates in the neighboring sites. In terms of the scattering length $a$, the trapping frequency $\omega_{L}$ in every site due to the optical lattice, and the characteristic length scale $l_{L}$ $=\sqrt{\hbar / m \omega_{L}}$, the interaction strength is given by

$$
U_{2 D}=4 \sqrt{\frac{\pi}{2}}\left(\frac{a}{l_{L}}\right) .
$$

Moreover, approximating the lattice potential near its maximum by an upside-down parabolic potential we obtain for the Josephson coupling

$$
J=\frac{1}{8 \pi^{2}}\left(\frac{\omega_{L}}{\omega_{r}}\right)^{2}\left(\frac{\lambda}{l_{r}}\right)^{2}\left[\frac{\pi^{2}}{4}-1\right] e^{-\left(\lambda / 4 l_{L}\right)^{2}},
$$

where $\lambda$ is the wavelength of the laser beams creating the optical lattice.

We use a variational approach to study the sound and the transverse breathing modes of the condensate. For this purpose we use the Gaussian ansatz

$$
\begin{aligned}
\Phi_{n}(x, y, t)= & \sqrt{\frac{N\left[B_{0}+\epsilon_{n}^{\prime}(t)\right]\left[1+\delta_{n}(t)\right]}{\pi}} \\
& \times \exp \left(-\frac{\left[B_{0}+\epsilon_{n}(t)\right]\left(x^{2}+y^{2}\right)}{2}+i \nu_{n}(t)\right)
\end{aligned}
$$

for the two-dimensional wave function at site $n$. The variational parameters $\epsilon_{n}=\epsilon_{n}^{\prime}+i \epsilon_{n}^{\prime \prime}$ are complex and describe the amplitude of the breathing mode, whereas $\delta_{n}$ and $\nu_{n}$ are the relative number fluctuation and the global phase at site $n$, respectively. Furthermore, $N$ is the equilibrium number of atoms at the site and $B_{0}$ gives the equilibrium size of the two-dimensional condensate. The latter is obtained by minimizing the equilibrium energy functional.

Using this ansatz we can calculate the energy functional. We find that in equilibrium the condensate size parameter $B_{0}$ is given by

$$
B_{0}=\frac{1}{\sqrt{1+2 U}}
$$

where, for convenience, we defined the strength of the interaction as

$$
U=\frac{N}{\sqrt{2 \pi}}\left(\frac{a}{l_{L}}\right) .
$$

In addition, the chemical potential is given by

$$
\mu=\frac{3}{2 B_{0}}-\frac{B_{0}}{2}-2 J
$$

because this choice for the chemical potential removes the terms linear in $\delta_{n}$ from the energy functional. 
As we are interested in the collective modes we must expand the Lagrangian $L=T-E$ up to second order in the variational parameters. Due to the technical simplicity of the number and global phase fluctuations we choose, for the time being, to treat them exactly and only expand in $\epsilon_{n}$. It then turns out that the contribution from the time-derivative term in the Lagrangian is

$$
\begin{aligned}
T / N & =\frac{i}{2} \sum_{n} \int d^{2} r\left[\Phi_{n}^{*} \frac{\partial \Phi_{n}}{\partial t}-\Phi_{n} \frac{\partial \Phi_{n}^{*}}{\partial t}\right] \\
& =\sum_{n}\left(1+\delta_{n}\right)\left[-\dot{\nu}_{n}+\frac{\dot{\epsilon}_{n}^{\prime \prime}}{2 B_{0}}\left(1-\frac{\epsilon_{n}^{\prime}}{B_{0}}\right)\right],
\end{aligned}
$$

whereas the energy functional becomes

$$
\begin{aligned}
E / N= & H_{J}+\sum_{n}\left\{\left(\frac{1+\delta_{n}}{2 B_{0}}\right)\left(1-\frac{\epsilon_{n}^{\prime}}{B_{0}}\right)+\left(\frac{\epsilon_{n}^{\prime}}{B_{0}}\right)^{2}+\frac{B_{0}}{2}\left(1+\delta_{n}\right)\right. \\
& \times\left[1+\frac{\epsilon_{n}^{\prime}}{B_{0}}+\left(\frac{\epsilon_{n}^{\prime \prime}}{B_{0}}\right)^{2}\right]+U B_{0}\left(1+\delta_{n}\right)^{2}\left(1+\frac{\epsilon_{n}^{\prime}}{B_{0}}\right) \\
& \left.-\mu N\left(1+\delta_{n}\right)\right\} .
\end{aligned}
$$

In Eq. (9) we have explicitly split off the contribution from the Josephson coupling,

$$
H_{J}=-J \sum_{\langle n, m\rangle}\left[\cos \left(\nu_{n}-\nu_{m}\right) I_{n m}^{\prime}-\sin \left(\nu_{n}-\nu_{m}\right) I_{n m}^{\prime \prime}\right]
$$

where $I_{m n}=\int d^{2} r \Phi_{m}^{*}(x, y) \Phi_{n}(x, y)$ is the overlap integral. Expanding the overlap integral up to second order, now also in the number and global phase fluctuations, we readily obtain

$$
\begin{aligned}
H_{J}= & \frac{J}{8 B_{0}^{2}} \sum_{\langle n, m\rangle}\left[\left(\epsilon_{n}^{\prime}-\epsilon_{m}^{\prime}\right)^{2}+2\left(\epsilon_{n}^{\prime \prime}-\epsilon_{m}^{\prime \prime}\right)^{2}+4 B_{0}^{2}\left(\nu_{n}-\nu_{m}\right)^{2}\right. \\
& \left.+B_{0}^{2}\left(\delta_{n}-\delta_{m}\right)^{2}-4 B_{0}\left(\nu_{n}-\nu_{m}\right)\left(\epsilon_{n}^{\prime \prime}-\epsilon_{m}^{\prime \prime}\right)\right]
\end{aligned}
$$

From this expression it is clear how the global phase fluctuations are coupled to the transverse breathing mode. Technically this is due to the imaginary part of the overlap integral which has a contribution linear in $\left(\epsilon_{n}^{\prime \prime}-\epsilon_{m}^{\prime \prime}\right)$. Such a contribution does not exist, for example, for the quadrupole modes which preserve the condensate volume. Therefore, quadrupole modes will not be affected by the presence of the sound mode.

In the above result for the Josephson energy $H_{J}$ we expanded also in terms of the number $\delta_{n}$ and global phase $\nu_{n}$ fluctuations. In the problem we are focusing on here these fluctuations are small and the above procedure is justified. In principle, however, number and global phase fluctuations can also be included exactly. By including them exactly we can also capture the physics of modulational and dynamical instabilities in an optical lattice [25-27], but this is outside the scope of the present paper.

\section{EQUATIONS OF MOTION AND THE EIGENMODES}

The results obtained in the preceding section enable us to derive the linearized Euler-Lagrange equations of motion for the variational parameters. They read

$$
\begin{gathered}
\frac{\partial \epsilon_{n}^{\prime}}{\partial t}=2 B_{0} \epsilon_{n}^{\prime \prime}+J \sum_{\langle n, m\rangle}\left(\epsilon_{n}^{\prime \prime}-\epsilon_{m}^{\prime \prime}\right), \\
\frac{\partial \epsilon_{n}^{\prime \prime}}{\partial t}=-\frac{2}{B_{0}} \epsilon_{n}^{\prime}-2 U B_{0}^{2} \delta_{n}-J \sum_{\langle n, m\rangle}\left(\epsilon_{n}^{\prime}-\epsilon_{m}^{\prime}\right), \\
\frac{\partial \nu_{n}}{\partial t}=-(1+3 U) \epsilon_{n}^{\prime}-3 B_{0} U \delta_{n} \\
-\frac{J}{2} \sum_{\langle n, m\rangle}\left[\left(\delta_{n}-\delta_{m}\right)+\left(\frac{\epsilon_{n}^{\prime}-\epsilon_{m}^{\prime}}{B_{0}}\right)\right],
\end{gathered}
$$

and

$$
\frac{\partial \delta_{n}}{\partial t}=2 J \sum_{\langle n, m\rangle}\left[\left(\nu_{n}-\nu_{m}\right)-\frac{\left(\epsilon_{n}^{\prime \prime}-\epsilon_{m}^{\prime \prime}\right)}{2 B_{0}}\right] .
$$

These equations of motion can also be written in a more formal way as

$$
\begin{gathered}
\frac{\partial \epsilon_{n}^{\prime}}{\partial t}=2 B_{0}^{2}\left(\frac{\partial E}{\partial \epsilon_{n}^{\prime \prime}}\right)+B_{0}\left(\frac{\partial E}{\partial \nu_{n}}\right), \\
\frac{\partial \epsilon_{n}^{\prime \prime}}{\partial t}=-2 B_{0}^{2}\left(\frac{\partial E}{\partial \epsilon_{n}^{\prime}}\right), \\
\frac{\partial \nu_{n}}{\partial t}=-\left(\frac{\partial E}{\partial \delta_{n}}\right)-B_{0}\left(\frac{\partial E}{\partial \epsilon_{n}^{\prime}}\right),
\end{gathered}
$$

and

$$
\frac{\partial \delta_{n}}{\partial t}=\frac{\partial E}{\partial \nu_{n}}
$$

While the first form of the equations is eventually needed in the actual calculations, the second way of writing the equations provides some additional insight. In particular, by inspecting Eqs. (16)-(18) it becomes clear how the global phases influence the behavior of the breathing mode through the term $B_{0}\left(\partial E / \partial \nu_{n}\right)$ in Eq. (16), and how the breathing mode influences the dynamics of the global phase through the term $-B_{0}\left(\partial E / \partial \epsilon_{n}^{\prime}\right)$ in Eq. (18). Without these terms we could treat the condensate density fluctuations independently from the transverse breathing mode.

The four first-order differential equations, Eqs. (12)-(15), for the variational parameters can be cast into two coupled second-order differential equations for $\epsilon_{n}^{\prime}$ and $\delta_{n}$. We are looking for solutions of the type $\epsilon_{n}^{\prime}=\epsilon_{k}^{\prime}(t) \sin (n k \lambda / 2)$ and $\delta_{n}=\delta_{k}(t) \sin (n k \lambda / 2)$. By inserting these into the equations of motion we obtain 


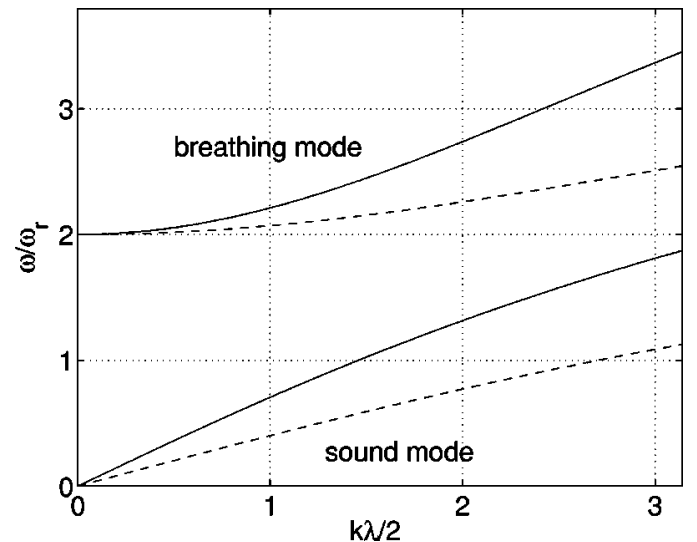

FIG. 1. Eigenmode frequencies of Eqs. (20) and (21) as a function of momentum, when $J=0.1, U=100$ (solid line), and $U=10$ (dashed line).

$$
\begin{aligned}
\frac{d^{2} \epsilon_{k}^{\prime}}{d t^{2}}= & -\left(2 B_{0}+2 J(k)\right)\left(\frac{2}{B_{0}}+2 J(k)\right) \epsilon_{k}^{\prime} \\
& +\left(B_{0}^{2}-1\right)\left[2 B_{0}+2 J(k)\right] \delta_{k}
\end{aligned}
$$

and

$$
\begin{aligned}
\frac{d^{2} \delta_{k}}{d t^{2}}= & 4 J(k)\left(B_{0}-\frac{1}{B_{0}}-J(k)\right) \delta_{k}-\frac{2 J(k)}{B_{0}}\left(3-3 B_{0}^{2}\right. \\
& \left.-\frac{2}{B_{0}}\right) \epsilon_{k}^{\prime},
\end{aligned}
$$

where $J(k)=J[1-\cos (k \lambda / 2)]$. The two eigensolutions of these equations correspond to the longitudinal sound mode and the transverse breathing mode. These equations of motion and their solutions are the main results of this paper.

In Fig. 1 we show a typical behavior of both the sound mode and the transverse breathing-mode frequencies as a function of momentum $k$ for two different values of the interaction strength. This figure demonstrates how the small $k$ behavior of the sound modes is linear whereas that of the breathing mode is quadratic. It also shows that both modes become stiffer as the strength of the interactions is increased. We devote the following two sections to the discussion of both modes separately.

\section{A. The longitudinal sound mode}

Theories of bosons in a lattice that reduce the problem to that of a global phase and an atom number in each site can be used to solve the dispersion relation $\omega_{S}(k)$ of the sound mode. We refer to these theories as phase-only theories. At small values of the momenta, the dispersion relation of the sound mode is linear in momentum, i.e., $\omega_{S}(k)=c_{0} k$. By using the ansatz (4), but removing the breathing modes, we find the phase-only sound velocity

$$
c_{0}=\lambda \sqrt{J B_{0} U}=\lambda\left(\frac{J U}{\sqrt{1+2 U}}\right)^{1 / 2} .
$$

This agrees with the results of Refs. [16-21].

However, this result is changed when the transverse breathing mode is taken into account. Using the unified description of sound and breathing modes that we have presented in this paper, we obtain a different sound velocity, namely,

$$
c=\lambda \sqrt{\frac{J}{8}\left(\frac{3}{B_{0}}-2 B_{0}-B_{0}^{3}\right)}=\lambda \sqrt{\frac{J U(2+3 U)}{2(1+2 U)^{3 / 2}}} .
$$

To obtain this simple expression we ignored contributions proportional to $J^{2}$ as these are in general very small.

In the noninteracting limit the sound velocity calculated from the phase-only theory coincides with the real sound velocity, but the relative shift $c / c_{0}-1$ becomes more pronounced with increasing interaction strength. In particular, the real sound velocity is smaller than the one predicted by the phase-only theories. Asymptotically we obtain in the limit of strong interactions $\lim _{U \rightarrow \infty}\left(c / c_{0}-1\right)=\sqrt{3 / 4}-1$ $\approx-0.13$. While this shift is not enormous, it is still large enough to be kept in mind when quantitative results are required. Intuitively, the reduction of the real sound velocity due to the transverse breathing mode is expected, since the transverse degree of freedom makes the condensate less stiff. Rather than all the energy of the density disturbance being pushed forward in the longitudinal direction, some of the excess energy is lost in exciting the transverse degree of freedom.

It is interesting to observe that while a "pure" breathing mode, unaffected by the global phase dynamics, is possible when all the sites are breathing in phase, a pure sound mode is never possible. The sound mode relies on global phase differences and number fluctuations between sites, and these will inevitably couple into the transverse breathing mode.

\section{B. The transverse breathing mode}

At small values of momenta the transverse breathing mode in the absence of sound mode has a dispersion relation [21]

$$
\omega_{0}=2+\frac{J \lambda^{2}}{8}\left(B_{0}+\frac{2}{B_{0}}\right) k^{2} .
$$

However, in line with our previous result for the sound mode, Eqs. (20) and (21) predict a different behavior for the term proportional to $k^{2}$; namely, we have

$$
\omega_{B}=2+\frac{J \lambda^{2}}{16}\left(B_{0}+\frac{5}{2 B_{0}}+\frac{B_{0}^{2}}{2}\right) k^{2},
$$

where we again ignored contributions proportional to $J^{2}$.

The breathing-mode dispersion relations can be given in terms of effective masses $m_{0}^{*}$ and $m_{B}^{*}$ for the transverse breathing mode as $\omega_{0}=2+k^{2} / 2 m_{0}^{*}$ and $\omega_{B}=2+k^{2} / 2 m_{B}^{*}$. The relative shift 
(a)

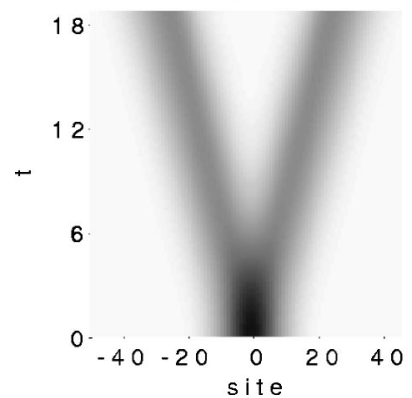

(b)

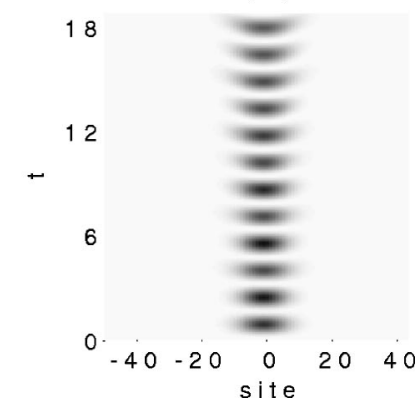

FIG. 2. The time evolution of (a) the density disturbance $\delta_{n}(t)$ and (b) the transverse breathing-mode amplitude $\left|\epsilon_{n}(t)\right|^{2}$. We used $J=0.1, U=100$, and the number of sites was 101 . The initial state had a Gaussian density disturbance $\delta_{n}(t=0)$ $=-0.1 \exp \left[-(n / 10)^{2}\right]$ and the unit of time is $1 / \omega_{r}$. In the figure dark color indicates the disturbance. It can be seen how the initial density dip splits into two dips propagating into opposite directions, whereas at this time scale the breathing mode remains well localized around the location of the initial density disturbance.

$$
\frac{m_{B}^{*}-m_{0}^{*}}{m_{0}^{*}}=\frac{3+2 B_{0}^{2}-B_{0}^{3}}{5+2 B_{0}^{2}+B_{0}^{3}}=\frac{6 U \sqrt{1+2 U}+5 \sqrt{1+2 U}-1}{10 U \sqrt{1+2 U}+7 \sqrt{1+2 U}+1}
$$

in the effective mass is always quite large and quickly approaches an asymptotic value $3 / 5$ as the strength of the interaction increases. This shift, therefore, must be included in making quantitative predictions for upcoming experiments.

\section{TIME EVOLUTION OF THE COUPLED SYSTEM}

Earlier in this paper we solved the equations of motion for the sound and the transverse breathing modes by assuming plane-wave solutions. This enabled us to obtain analytic solutions for the dispersion relations, but our theory can be used to solve also more complicated problems. In this section we demonstrate this by solving the coupled dynamics of the density fluctuations and the transverse breathing mode, when the initial state of the condensate has a density dip. This problem is interesting since the earlier experiments on a condensate sound mode first created a density dip and then tracked the evolution of the condensate density $[3,4]$. In this case calculating the time evolution of the coupled system of the sound mode and the transverse breathing mode is too complicated to be attacked analytically, but the problem can be readily tackled numerically.

In Fig. 2 we show an example of the typical time evolution. In this figure we prepare the system with a Gaussian density disturbance $\delta_{n}(t=0)=-0.1 \exp \left[-(n / 10)^{2}\right]$ and then let it evolve. The density minimum splits into two parts propagating into opposite directions. We find that the numerically calculated propagation velocity of the density disturbance is in good agreement with our analytical result for the sound velocity in Eq. (23) and very different from the phase-only result in Eq. (22). The excess energy of the density dip excites the transverse breathing mode that remains well localized in the center of the lattice and only slowly
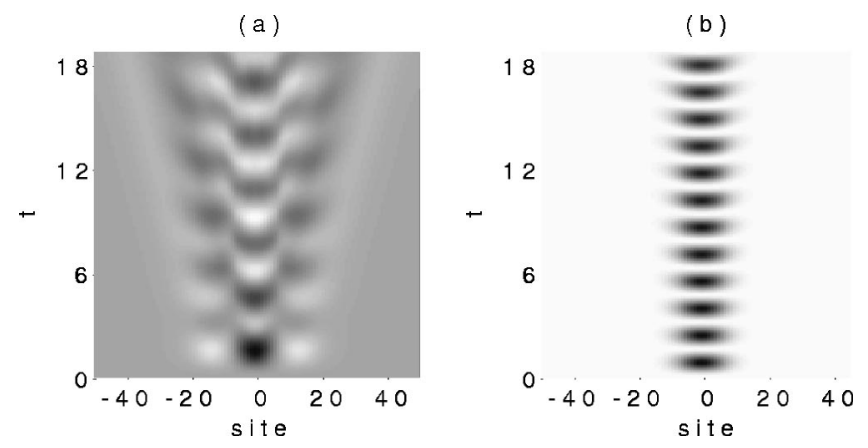

FIG. 3. The time evolution of (a) the density disturbance $\delta_{n}(t)$ and (b) the transverse breathing-mode amplitude $\left|\epsilon_{n}(t)\right|^{2}$. We used $J=0.1, U=100$, and the number of sites was 101 . The initial state had a Gaussian transverse breathing-mode disturbance $\epsilon_{n}(t=0)$ $=B_{0} / 2 \exp \left[-(n / 10)^{2}\right]$. This choice corresponds to the fractional change of $-25 \%$ of the condensate size in the center of the lattice. The unit of time is $1 / \omega_{r}$. In (a) dark color indicates a region of low density. In (b) it would seem that the breathing-mode amplitude is initially zero. This is a result of plotting the amplitude squared $\left|\epsilon_{n}(t)\right|^{2}$ as opposed to plotting just the real part $\epsilon_{n}^{\prime}(t)$. Typically the imaginary part of the breathing-mode amplitude has a larger magnitude and therefore dominates in the amplitude squared plot.

spreads out further. This slow spreading of the breathing mode is hardly visible at the relatively short time scale of the figure.

In Fig. 3 we demonstrate the "inverse" problem of an initial state that has a localized transverse breathing-mode disturbance and a homogeneous density distribution. The time evolution of the density distribution is then more complicated, but the time evolution of the breathing-mode amplitude is similar to that in Fig. 2. In Fig. 3 the fractional change of the condensate size was $-25 \%$ in the center of the lattice and such a deformation is quite large. Despite this strong deformation, the magnitude of the density disturbance remains small, below $1 \%$. Therefore, under these conditions it would be difficult to image the density disturbance experimentally.

\section{SUMMARY AND CONCLUSIONS}

We have presented a unified theory of the sound propagation and the transverse breathing mode of a Bose-Einstein condensate in a one-dimensional optical lattice. Using a variational ansatz we calculated the dispersion relations of both modes, and found out that the dispersion relations are quite strongly modified by the coupling between the sound and the transverse breathing modes. These changes are large enough that they should be included when making quantitative predictions for experiments. In principle, the sound mode is coupled not only to the transverse breathing mode but also to modes with higher energy that have the same symmetry. In this paper we have ignored such higher-order effects, since the overlap with the transverse breathing mode is the largest and therefore the coupling to this mode dominates.

In principle, the theories studying two weakly coupled condensates $[28-30]$ would also be influenced by the mecha- 
nism we have discussed in this paper. This makes the study of the phase dynamics of the weakly coupled condensates in the presence of the transverse degrees of freedom an interesting topic for further research.

When the sound mode in an ordinary elongated BoseEinstein condensate is excited, by using, for example, a bluedetuned laser beam as in the experiments of Andrews et al. $[3,4]$, the transverse profile of the condensate is modified close to the laser beam. After removing the laser beam, the density dip starts propagating with the sound velocity. However, we have seen that in principle in addition to this also a surface disturbance starts propagating in the condensate. This is analogous to the problem we have studied in this paper. In contrast with the Bose-Einstein condensate in an optical lattice, in a cigar-shaped three-dimensional Bose-Einstein condensate the influence of the transverse degrees of freedom is expected to be small, and theories ignoring them [6-8] are indeed in agreement with the experiments [3-5].

Our theory can also be used to study how the transverse breathing mode excites density modulations of the condensate in an optical lattice. In this paper we gave one numerical example along these lines, but when the transverse breathing-mode amplitude is very large or strongly modulated, we expect that nonlinearities will play an important role. Under such conditions instabilities might arise, revealing possibilities for studying nonlinear matter wave dynamics.

\section{ACKNOWLEDGMENTS}

This work was supported by the Stichting voor Fundamenteel Onderzoek der Materie (FOM) and by the Nederlandse Organisatie voor Wetenschaplijk Onderzoek (NWO).
[1] N. Bogoliubov, J. Phys. (USSR) 11, 23 (1947).

[2] P. Noziéres and D. Pines, The Theory of Quantum Liquids, Superfluid Bose Liquids Vol. II (Addison-Wesley, New-York, 1990).

[3] M.R. Andrews, D.M. Kurn, H.-J. Miesner, D.S. Durfee, C.G. Townsend, S. Inouye, and W. Ketterle, Phys. Rev. Lett. 79, 553 (1997).

[4] M.R. Andrews, D.M. Kurn, H.-J. Miesner, D.S. Durfee, C.G. Townsend, S. Inouye, and W. Ketterle, Phys. Rev. Lett. 80, 2967 (1998).

[5] D.M. Stamper-Kurn, A.P. Chikkatur, A. Görlitz, S. Inouye, S. Gupta, D.E. Pritchard, and W. Ketterle, Phys. Rev. Lett. 83, 2876 (1999).

[6] E. Zaremba, Phys. Rev. A 57, 518 (1998).

[7] G.M. Kavoulakis and C.J. Pethick, Phys. Rev. A 58, 1563 (1998).

[8] S. Stringari, Phys. Rev. A 58, 2385 (1998).

[9] M. Cristiani, O. Morsch, J.H. Müller, D. Ciampini, and E. Arimondo, Phys. Rev. A 65, 063612 (2002).

[10] C. Orzel, A.K. Tuchman, M.L. Fenselau, M. Yasuda, and M.A. Kasevich, Science 291, 2386 (2001).

[11] M. Greiner, O. Mandel, T.W. Hänsch, and I. Bloch, Nature (London) 419, 51 (2002).

[12] F.S. Cataliotti, S. Burger, C. Fort, P. Maddaloni, F. Minardi, A. Trombettoni, A. Smerzi, and M. Inguscio, Science 293, 843 (2001).

[13] S. Burger, F.S. Cataliotti, C. Fort, F. Minardi, M. Inguscio, M.L. Chiofalo, and M.P. Tosi, Phys. Rev. Lett. 86, 4447 (2001).
[14] D. Jaksch, C. Bruder, J. Cirac, C.W. Gardiner, and P. Zoller, Phys. Rev. Lett. 81, 3108 (1998).

[15] M. Greiner, O. Mandel, T. Esslinger, T.W. Hänsch, and I. Bloch, Nature (London) 415, 39 (2002).

[16] D. van Oosten, P. van der Straten, and H.T.C. Stoof, Phys. Rev. A 63, 053601 (2001).

[17] K. Berg-Sørensen and K. Mølmer, Phys. Rev. A 58, 1480 (1998).

[18] J. Javanainen, Phys. Rev. A 60, 4902 (1999).

[19] M. Krämer, L. Pitaevskii, and S. Stringari, Phys. Rev. Lett. 88, 180404 (2002).

[20] M. Machholm, C.J. Pethick, and H. Smith, Phys. Rev. A 67, 053613 (2003).

[21] J.-P. Martikainen and H. Stoof, Phys. Rev. A 68, 013610 (2003).

[22] E. Taylor and E. Zaremba, Phys. Rev. A 68, 053611 (2003).

[23] F. Meier and W. Zwerger, Phys. Rev. A 64, 033610 (2001).

[24] I. Zapata, F. Sols, and A.J. Leggett, Phys. Rev. A 67, 021603 (2003).

[25] B. Wu and Q. Niu, Phys. Rev. A 64, 061603 (2001).

[26] V.V. Konotop and M. Salerno, Phys. Rev. A 65, 021602 (2002).

[27] B.B. Baizakov, V.V. Konotop, and M. Salerno, J. Phys. B 35, 5105 (2002).

[28] A. Smerzi, S. Fantoni, S. Giovanazzi, and S.R. Shenoy, Phys. Rev. Lett. 79, 4950 (1997).

[29] I. Zapata, F. Sols, and A.J. Leggett, Phys. Rev. A 57, R28 (1998).

[30] S. Raghavan, A. Smerzi, S. Fantoni, and S.R. Shenoy, Phys. Rev. A 59, 620 (1999). 